\title{
The natural history of epiphora in childhood
}

Abstract

Purpose To investigate the incidence and aetiology of epiphora in a population of 7-year-old children and determine whether a conservative management policy for congenital nasolacrimal duct obstruction (CNLDO) results in excess lacrimal dysfunction in later childhood.

Methods A postal questionnaire was sent to the parents of a large cohort of 7-year-old children previously investigated to determine the incidence and natural progression of CNLDO. They were asked about the presence of epiphora, and its relationship to atopic disease and the presence of upper respiratory tract infections (URTI). Information on epiphora had also been gathered from the same cohort at age $3 \frac{1}{2}$ years at a routine examination. Data were compared with those for epiphora in infancy in the same cohort. Results The incidence of epiphora at $3 \frac{1}{2}$ years was $5.5 \%$, and 7 years $7.7 \%$. At age 7 years $70 \%$ of cases were related to atopic disease or URTI. CNLDO was not significantly related to epiphora in later childhood ( $p=0.000032)$. Conclusions A policy of delaying nasolacrimal probing in CNLDO until after the age of 1 year does not result in a detectable excess of lacrimal dysfunction in later childhood, when epiphora is more likely to be related to atopic disease or upper respiratory tract infection.

Key words Epiphora, Paediatrics, Resolution, Atopy, Incidence, Aetiology

Epiphora is common in infancy and usually due to congenital nasolacrimal duct obstruction (CNLDO). Many authors have advised that this condition should be promptly treated with probing of the nasolacrimal duct, while others, recognising the high rate of spontaneous resolution, have advised delay in surgical intervention for up to 18 months. ${ }^{1-3}$ One of the reasons given for early probing has been the risk that chronic infection associated with delayed treatment might cause a higher incidence of lacrimal stenosis in later childhood. ${ }^{4-6}$ At present this possibility cannot be excluded as there is little information in the literature on the incidence and aetiology of epiphora after the age of 24 months, although atopic disease (hay fever, asthma, eczema) is thought to be causal. ${ }^{7}$

The aim of this study was to establish the frequency and aetiology of lacrimal symptoms in later childhood and to determine whether there was any relationship with previous CNLDO where treatment had been delayed to allow for spontaneous resolution.

\section{Subjects and method}

The population studied was a cohort consisting of all children born in 1988 and living in the Tayside Health Board Area. This cohort had originally been studied during their first year of life, to determine population incidence and the natural course of CNLDO. ${ }^{1}$ We have now been able to restudy the same cohort on two further occasions, at $3 \frac{1}{2}$ and 7 years of age.

At age $3 \frac{1}{2}$ years all the children in the Tayside area are offered a developmental surveillance examination, undertaken by community paediatricians. For those children in the study cohort additional enquiry was made for lacrimal symptoms using a standard proforma. The parents were asked about the presence of 'significant' epiphora in the preceding 2 years in their child, significant being defined as any episode continuing for more than 1 week. They were also asked about the characteristics of the epiphora: whether it was intermittent or constant, and its relationship to colds.

A final survey of the cohort was undertaken by postal questionnaire when the children were 7 years of age. The questionnaire asked whether the child had suffered 'significant' watering or stickiness in the last year. If the answer was affirmative the parents were asked:

1. If the symptoms were related to allergic disease (hay fever/asthma)

2. If the symptoms only occurred in the presence of colds

3. Whether topical antibiotics had been used in the past year

4. If the child was currently symptomatic

5. If this had been a problem in the long term

6. If they would like an appointment for their child
R. Maini

C.J. MacEwen J.D.H. Young Department of Ophthalmology Ninewells Hospital and Medical School Dundee DD1 9SY, UK

Mr R. Maini

Department of Ophthalmology West Suffolk Hospital Bury St Edmunds Suffolk IP3 2QZ, UK 
Those parents who requested it were sent an appointment for further investigation of the symptoms. Using a set protocol the patients were examined, looking in particular for any abnormal lacrimal function with a fluorescein dye disappearance test (FDT) ${ }^{8}$ and for any other ocular pathology or anatomical abnormality that would explain the symptoms. Telephone contact was attempted with all the non-attenders. The data were then compared with those available for the same cohort in their first year of life.

\section{Results}

An incidence of $20.1 \%$ for CNLDO with $96 \%$ of cases resolving by the age of 1 year had already been determined for the cohort. ${ }^{1}$

At $3 \frac{1}{2}$ years data were obtained on 2389 of 4792 $(49.9 \%)$ of the cohort. Of these 2389,131 (5.5\%) had had significant epiphora in the preceding 2 years and 97 of $131(74 \%)$ of these cases were new, i.e. occurring in children who had never suffered CNLDO.

Of the 131 children with significant epiphora, in 72 $(54.9 \%)$ the problem only occurred in the presence of colds. In 126 of the 131 (96.2\%) the parents felt the watering was intermittent, and in 5 of the $131(3.8 \%)$ that it was constant.

A total of 4990 questionnaires were sent out to the parents of all the children in the cohort at age 7 years; 2830 completed questionnaires were returned, a response rate of $56.9 \%$. Parents of 219 children confirmed significant epiphora had occurred in the last year. The incidence of epiphora in our population of 7-year-old children was therefore $7.7 \%$.

Parents felt that the epiphora was related to allergies in 79 of $219(36.1 \%)$; only occurred in the presence of colds in 78 of 219 (35.6\%); was a long-term problem in 40 of $219(18.3 \%)$ and that 55 of $219(25.1 \%)$ children were currently symptomatic. Topical antibiotics had been used in the preceding year in 110 of 219 (50.2\%).

Six of the 219 patients had already had ophthalmic investigation for epiphora, which in all cases had developed after the first year of life. Two had undergone unilateral probing with apparent resolution of their symptoms; one currently had nasolacrimal tubes in situ after bilateral probing; one had had a diagnosis of meibomianitis; and one mother felt the symptoms had resolved with the treatment of her child's amblyopia.

Table 1. Characteristics of the sample examined $(n=16)$

\begin{tabular}{ll}
\hline Finding & No. \\
\hline Allergic conjunctivitis & 6 \\
Squamous blepharitis & 6 \\
Epiblepharon & 1 \\
Abnormal FDT & 1 \\
Follicular conjunctivitis & 1 \\
No abnormality detected & 1
\end{tabular}

FDT, fluorescein dye disappearance test.
Table 2. Age of resolution of CNLDO in those with epiphora at age 7 years

\begin{tabular}{lc}
\hline Patient no. & Age at resolution \\
\hline 1 & 10 days \\
2 & 14 days \\
3 & 3 weeks \\
4 & 6 weeks \\
5 & 1 month \\
6 & 1 month \\
7 & 2 months \\
8 & 3 months \\
9 & 3 months \\
10 & 6 months \\
11 & 7 months \\
12 & 10 months \\
\hline
\end{tabular}

There were 25 requests by parents of symptomatic children for a clinic appointment, but only 16 of these children attended. The results of these examinations are detailed in Table 1.

Telephone contact was attempted with the 9 nonattenders' parents. None of the parents contacted wished a further appointment and 4 of the children had had spontaneous resolution of their symptoms; in one case the family doctor had made a presumptive diagnosis of viral conjunctivitis and in another the mother felt the problem had arisen when her son had started swimming lessons - this had coincided with the arrival of the questionnaire!

Comparative analysis with data already available for the same cohort revealed that of the 219 children with epiphora at age 7 years only 17 had suffered CNLDO. A further 12 of the 219 had admitted to epiphora at the $3 \frac{1}{2}$ year survey, whilst only 5 of 219 had had CNLDO and epiphora at the $3 \frac{1}{2}$ year survey. This meant that 12 children with CNLDO had spontaneous resolution of the epiphora by the age of 1 year (with $75 \%$ of these resolving by 3 months of age) with a subsequent recurrence of symptoms in later childhood (Table 2).

Yates' corrected chi-squared analysis comparing the first year of life with the eighth revealed that epiphora in infancy is not significantly associated with epiphora in later childhood ( $p=0.000032)$ (Table 3).

\section{Discussion}

This is the first large population-based study to investigate the natural course of epiphora from birth to the eighth year of life. The response rate to the questionnaires of $56.9 \%(n=2830)$ should be considered in the light of the population turnover documented in Tayside. GP registration in Tayside in 1995 stood at

Table 3. The relationship between CNLDO and epiphora at age 7 years (chi-squared analysis). Figures are fractionated to ensure equivalence regarding response rates to the questionnaires

\begin{tabular}{lrrr}
\hline & CNLDO & No CNLDO & Total \\
\hline Epiphora at 7 years & 17 & 202 & 219 \\
No epiphora at 7 years & 505 & 2106 & 2611 \\
Total & 522 & 2308 & 2830 \\
\hline
\end{tabular}


410 322, with 10497 people leaving the area and 11536 coming in, giving an annual population turnover of approximately $2.5 \%,{ }^{9}$ implying a loss of $17.5 \%$ of the population over the 7 years between the two studies. Although the statistics for 7-year-olds were unavailable, extrapolation would imply only approximately $82.5 \%$ of the original cohort investigated by MacEwen and Young ${ }^{1}$ remained in the area, giving a 'true' response rate of $68.7 \%$.

This study has shown that CNLDO is not significantly associated with lacrimal symptoms in later childhood. At $3 \frac{1}{2}$ and 7 years of age watering is likely to be due to allergic disease or upper respiratory tract infections, these aetiologies accounting for $70 \%$ of the symptomatic children in this study. Interestingly over half the symptomatic children had used topical antibiotics for the condition, implying a fairly low threshold for prescription in the primary care setting.

Epiphora in childhood is common in the first few months of life $(20 \%)$, with a high rate of spontaneous resolution during the first year, ${ }^{1}$ and we have now shown this is followed by an increased incidence in later childhood. We consider this pattern is consistent with the different aetiologies we have identified in the two age groups.

Only 3 of the symptomatic children at 7 years had had therapeutic probings, and all had developed symptoms after the age of 1 year, implying a non-developmental cause for the lacrimal outflow obstruction - possibly secondary to an infective episode.

The sample examined was self-selected and small in number, as a result of the difficulty in getting patients to attend clinic. Parents tend to view the problem as minor in nature (especially if not associated with inflammation), and are unlikely to attend should the condition resolve spontaneously. However, the results we do have confirm that allergic disorders are a significant cause of symptoms, and surprisingly an equivalent number manifested squamous blepharitis.
This was characterised predominantly by scaling, rather than any significant meibomian dysfunction. Blepharitis in children is more usually associated with conjunctivitis. ${ }^{10}$ It is not possible to draw firm conclusions regarding this because of our small sample size, but the role of blepharitis in producing epiphora in children may merit further investigation.

Our findings in this study further reinforce the view that the management of choice for epiphora in infancy is to delay probing to beyond the first birthday in order to allow for the high rate of spontaneous resolution. We have now confirmed that this policy does not have a detrimental effect on nasolacrimal function in later childhood - any symptoms in this age group are likely to have alternative aetiology.

\section{References}

1. MacEwen CJ, Young JDH. Epiphora during the first year of life. Eye 1991;5:596-600.

2. Nelson LB, Calhoun JH, Menduke H. Medical management of congenital nasolacrimal duct obstruction. Ophthalmology 1985;92:1187-90.

3. Paul TO. Medical management of congenital nasolacrimal duct obstruction. J Pediatr 1985;22:68-70.

4. Katowitz JA, Welsh MG. Timing of initial probing and irrigation in congenital nasolacrimal duct obstruction. Ophthalmology 1987;94:698-705.

5. Ffookes OO. Dacryocystitis in infancy. Br J Ophthalmol 1962;46:422-34.

6. Kushner BJ. Congenital nasolacrimal duct obstruction. Arch Ophthalmol 1982;100:597-600.

7. Day S. The watery eye. In: Taylor D, editor. Paediatric ophthalmology. Oxford: Blackwell Scientific, 1990:682-4.

8. MacEwen CJ, Young, JDH. The fluorescein disappearance test: an evaluation of its use in infants. J Paediatric Ophthalmol Strabismus 1991;28:302-5.

9. Personal communication. Primary Care Division, Tayside Health Board, Maryfield House, Dundee, Scotland.

10. Pratap VB, Lal HB. Pattern of paediatric ocular problems in north India. Indian J Ophthalmol 1989;37:171-2. 